\title{
Verification of a theoretical model of tensor skin under water impact by considering the fluid-structure interaction
}

\author{
YanTing REN ${ }^{\mathrm{a}}$, XinMing QIU ${ }^{\mathrm{a}^{*}}$, T.X. YU ${ }^{\mathrm{b}, \mathrm{c}}$ \\ ${ }^{\mathrm{a}}$ Department of Engineering Mechanics, Tsinghua University, Beijing 100084, China \\ ${ }^{\mathrm{b}}$ Department of Mechanical Engineering, Hong Kong University of Science and Technology, Clear \\ Water Bay, Kowloon, Hong Kong \\ ${ }^{c}$ Y.K. Pao Chair Professor, ZhejiangUniversity, Hangzhou, China \\ * Corresponding author, 8610- 62772329, e-mail: qxm@tsinghua.edu.cn
}

\begin{abstract}
Tensor skin is a kind of composite sandwich structure developed to improve the helicopter's crashworthiness when impact with water. In our previous theoretical studies, by employing the rigid, perfectly plastic idealization and the pulse approximation method, the static and dynamic responses of tensor skin under uniform pressure loading were obtained.

In this study, a fluid-structure interaction simulation model of tensor skin impinging with water is established by FE method, in order to verify the previous theoretical analyses. It is found that the interaction pressure between the skin plate and water varies with the configuration of the plate. Compared with a solid plate, the pulses generated by a honeycomb-core sandwich plate and a tensor skin are more moderate. By studying the effect of water depth, the theoretical predictions of the tensor skin, as developed in our previous papers, are found valid for the deep water case.
\end{abstract}


1 From the comparison among the theoretical predictions, pulse loading FE model, and the

2 fluid-structure interaction FE model, the deviation of the theoretical prediction in the final

3 deflection is found to be about $10 \%$ compared with that obtained from the fluid-structure

4 interaction FE model. Thereby, the theoretical model and the pulse approximation method are

5 confirmed to have sufficient accuracy for the design of tensor skins.

\section{$6 \quad$ Keywords}

7 Tensor skin, Verifications, Theoretical model, Water impact, Energy absorption

8 


\section{Notations}

$3 \quad \rho$ is density

$4 E_{l}, E_{t}$ are the elastic modulus in the longitudinal and transverse directions, respectively

$5 \quad G$ is the shear modulus

6

$7 h_{1}, h_{2}, h_{3}$ are thicknesses of cover, carrying and tensor ply, respectively

$8 \quad H$ is the depth of water

$9 \quad I$ is momentum

$10 \quad k$ is the geometric parameter of tensor skin

$11 \quad l$ is a half of the beam length

$12 M_{P}$ is the fully plastic bend moment

$13 q$ is pressure applied on the beam

$14 q_{s}$ is the static critical pressure

$15 \quad \bar{q}_{s} \equiv q_{s} l^{2} / M_{P}$ is the normalized static critical pressure

$16 \tilde{q} \equiv \bar{q}-\bar{q}_{S}$ is the excess pressure

$17 t_{d}$ is impulse duration

$18 t_{f}$ is the total response time

$19 u$ is the central beam deflection

$20 \quad \bar{u} \equiv u / l \quad$ is the normalized central deflection

21

22 
1

\section{Introduction}

As a kind of sandwich structure comprised of cover plies, tensor plies and carrying plies, tensor skin is originally developed by NLR (European National Aerospace Laboratory) in order to improve the crashworthiness of composite helicopter structures subjected to water impact [1], as shown in Fig.1. The cover ply is the loading face; the carrying ply provides the structure stiffness; and the tensor ply provides the capability to unfold by forming plastic hinges, before it is stretched and fails, leading to an increase in the load bearing capability and energy absorption of the structure.

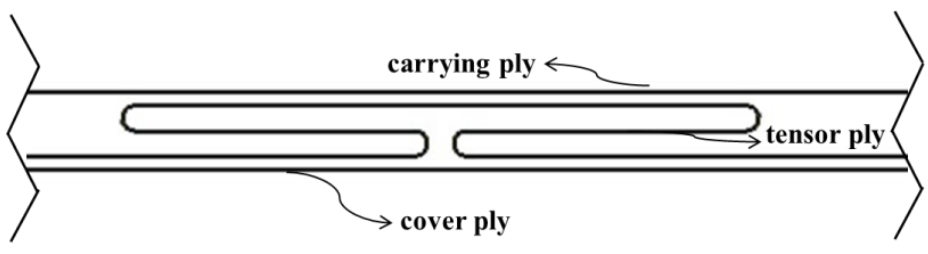

Fig.1 The schematic of a tensor skin

Under the loading of tension or bending, the deformation mechanism of the tensor skin strip was found to be unfolding by forming plastic hinges before the failure due to stretching $[2,3]$. The static and dynamic responses of tensor skin panels were compared with the honeycomb-core sandwich plates [4-7]. In the static transverse loading tests, the fully clamped panels were pushed by a blunt indenter that was perpendicular to the surface. Only 3-layer tensor skin panel was able to transfer sufficient running load to the substructure to initiate crushing. In the dynamic tests performed by dropping an impactor, large damage was found in the honeycomb-core sandwich, while the tensor skin managed to stop the impactor successfully, with broken faces and unfolded core. The failure behavior of the tensor skin was also calculated by using PAM-CRASH [8]. Compared with the above mentioned experimental results [4, 7-11], good agreements between the 
calculated and measured forces and displacement for tensor skin panels were found. Besides, three types of innovative Leading Edge structures that made of tensor skin were designed, and also tested by the impact of a 4-pound substitute bird [5]. The relative numerical studies by PAM-CRASH were given by Mi[12]. Both results confirmed the good performance of tensor skins in the anti-bird strike designs.

A theoretical model was proposed by the present authors [13] to analyze the static and dynamic responses of tensor skin, in which the rigid-perfectly plastic idealization was adopted. It was found that the tensor skin exhibits unique properties in its load-displacement relationship. That is, the load to initiate the plastic deformation is high, but the resistance decreases during the structure's deformation process. Furthermore, the pulse approximation method, initially proposed by Youngdahl, was confirmed to be applicable to this structure, tensor skin [13]. The main conclusions from the theoretical analysis will be reviewed in Section 2.

As well known, the dynamic response of a structure is an interaction process between the structure and the applied load pulse. However, in the theoretical approach, both the structure and the applied load have to be idealized in order to make the analysis feasible. For the tensor skin, the rigid-perfectly plastic material model was adopted in [14], and the effect of water impact on the structure was simplified as a triangular pulseapplied on the tensor skin [13]. In all the previous studies of the tensor skin, including experimental studies, numerical simulations, and theoretical modeling $[2,4,5,10,13,14]$, no fluid-structure interaction effect has been considered. Hereby, it is necessary to verify the existing theoretical model of the pressure loaded tensor skin with the fluid-structure interaction being taken into account.

In the present paper, in order to verify the previous theoretical model, the finite element 
simulations of the tensor skin under water impact are described, with the consideration of the fluid-structure interaction during the impact process. First, the theoretical analysis on the tensor skin under uniformly distributed pressure is reviewed in Section 2; and then the fluid-structure interactive FE models are described in Section 3; followed by Section 4, in which the dynamic responses of solid plate, tensor skin and honeycomb-core sandwich under water impacts are compared with one another; after a discussion on the effects of water depth in Section 5, the theoretical predictions of tensor skin are finally verified in Section 6.

\section{Review of the theoretical analysis of tensor skin}

A theoretical model of tensor skin was proposed in our previous paper[14], in which the total response of tensor skin is divided into three stages: an elastic deformation stage of the whole structure; an unfolding stage of the tensor ply; and a stretching stage of the tensor ply. At the beginning of response, elastic deformation takes place in the entire structure, which will end by the fracture of the cover and carrying plies made of brittle composite. The second stage is the unfolding of the tensor ply, which is simplified as rigid segments connected by plastic hinges. The third stage is the stretching of the tensor ply, which may be terminated by tension failure. Among the three stages, the unfolding stage is the main contributor to the energy absorption. Therefore, the theoretical analysis of tensor ply will be reviewed in the following.

\section{$18 \quad 2.1$ Static critical pressure}

In the unfolding stage, by adopting the rigid, perfectly plastic material assumption, a deformation mechanism with stationary plastic hinges was proposed[14], supposing a uniformly distributed pressure $q$ is applied on the tensor ply quasi-statically, as shown in Fig.2. Hereby, as 
5 variation in the energy dissipation $\delta D$. That is,

where $l$ is a half of the beam length and $M_{p}$ is the fully plastic bending moment.

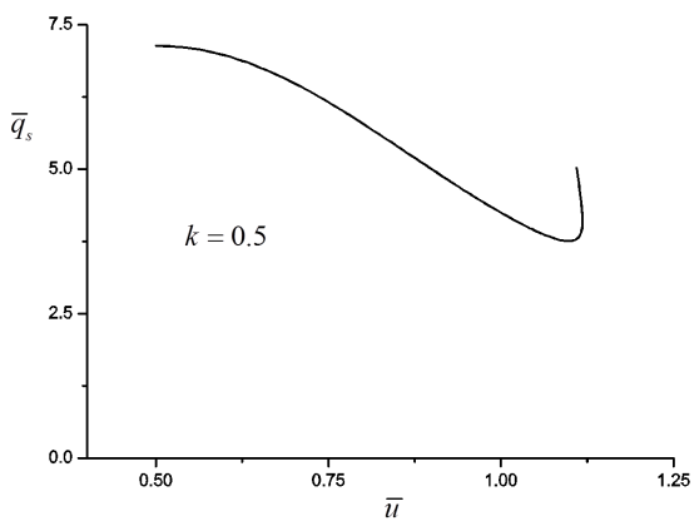

Fig.2 Tensor ply under uniformly distributed pressure in the unfolding stage [14]

From the energy conservation, the increase in the external work $\delta W$ should be equal to the Hence, the normalized static critical pressure is obtained as,

$$
\bar{q}_{s}(\alpha) \equiv q_{s}(\alpha) l^{2} / M_{p}
$$

11

Fig.3 The normalized static critical pressure versus the normalized central deflection $\bar{u}$ [14]
It is found that the static critical pressure $\bar{q}_{s}$ first decreases then increases with the increasing central deflection $\bar{u} \equiv u / l$, as demonstrated in Fig.3. The shape of the curve indicates a kind of softening effect; that is, the pressure to motivate the initial deformation is high, but the structure's resistance decreases as the deformation becomes larger. That is different from the 


\section{$2 \quad 2.2$ Dynamic response of tensor ply}

The dynamic response of tensor ply was also analyzed in [14]. Here, the static critical pressure $\bar{q}_{s}$ is regarded as a kind of structural friction. Therefore, the excess pressure defined as $\tilde{q} \equiv \bar{q}-\bar{q}_{s}$ will produce the acceleration or deceleration of the structure.

Suppose a constant pressure $\bar{q}>\bar{q}_{s 0}$ is applied on the tensor skin, here $\bar{q}_{s 0}$ is the normalized initial static critical pressure, which is given by Eq.(2) for $\alpha=0$. According to energy conservation,

$$
\dot{K}+\dot{D}=\dot{W}(q)
$$

where $\dot{K}$ is the rate of kinetic energy, $\dot{D}$ is the rate of plastic dissipation, and $\dot{W}(q)$ is the work rate of the pressure. Eq.(3) leads to a second order differential equation about $\alpha$. Hereby, the dynamic response could be obtained by solving this equation with specified initial conditions[14].

\subsection{Pulse approximation of water impact}

\section{Youngdahl[15] proposed that the response of a general pulse could be approximately} equivalent to that of a rectangular pulse impulse $I_{e}$ with an effective load $P_{e}$ and pulse duration $2 t_{\text {mean }}$, which are defined by:

$$
I_{e}=\int_{t_{y}}^{t_{f}} P(t) \cdot \mathrm{d} t, \quad P_{e}=I_{e} / 2 t_{\text {mean }}, t_{\text {mean }}=\frac{1}{I_{e}} \int_{t_{y}}^{t_{f}}\left(t-t_{y}\right) P(t) \cdot \mathrm{d} t
$$

where $P(t)$ is the non-negative pulse magnitude changing with time $t, t_{y}$ and $t_{f}$ are the time instants when plastic deformation begins and ends, respectively. The final deflection of the structure is determined by $I_{e}$ and $P_{e}$ for a general pulse load. Depended on the integral of the 
general pulse, both $I_{e}$ and $P_{e}$ are not very sensitive to the pulse shape.The applicability of this approximation method for eliminating the pulse shape effects was validated for beams, circular plates and cylindrical shells, etc.[16].

In paper[13], the applicability of the above pulse approximation method was further examined for tensor skin. Meanwhile, as illustrated in [17], for a blunt or flat structure impinging on a water surface, the impact pulse only lasts for a very short time duration, which is very similar to a triangular pulse. It is found in [14], on the other hand, that the dynamic response of a tensor skin to a triangular pulse is quite close to that to an equivalent rectangular pulse. Fig. 4 shows the triangular pulse and the equivalent rectangular pulse, and the difference between their final deflections is found to be smaller than $5 \%$. Here $\bar{I}=\bar{q} \bar{t}_{d}$ and $\bar{q}=1.3 \bar{q}_{s 0}, \bar{t}_{d} \equiv t_{d} / t_{0}=1$, where $t_{0}=0.2 s$.

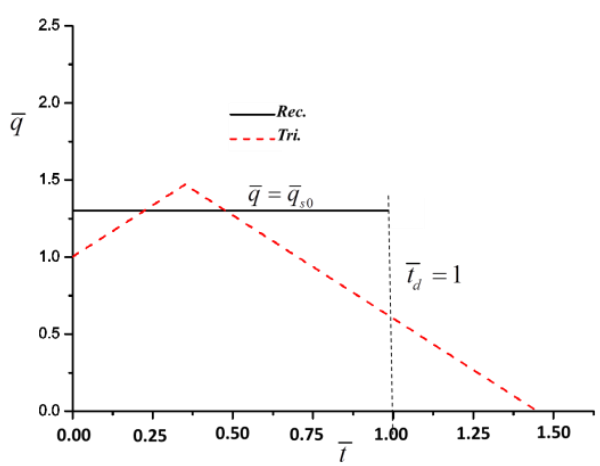

Fig.4 A triangular pulse and the equivalent rectangular pulse $\left(\bar{I}=1.3 \bar{q}_{s 0}, \bar{t}_{d}=1\right)$

\section{The fluid-structure interaction simulation model}

As mentioned in Introduction, none of the previous researchers has considered the effect of fluid-structure interaction, but this effect may provide a pressure pulse different from that adopted in the previous theoretical analysis. Therefore, by employing the commercial software PATRAN/DYTRAN, 3D simulation models are constructed in order to reveal the interaction effect 
1 between water and tensor skin. Totally 7500 quadrilateral shell elements are divided for the tension skin, and 9600 hexahedral solid elements (CHEXA in DYTRAN) are divided for the fluid

3

4

6 calculation is $10^{-8} \mathrm{~s}$.

\section{$7 \quad 3.1$ Models of three kinds of plates}

8

9

10

\begin{tabular}{cccccccc}
\hline \hline \multirow{2}{*}{ Ply } & Material & $\rho\left(\mathrm{Kg} / \mathrm{m}^{3}\right)$ & $E_{l}(\mathrm{MPa})$ & $E_{t}(\mathrm{MPa})$ & $G(\mathrm{MPa})$ & $\sigma_{\mathrm{Y}}(\mathrm{MPa})$ & $\varepsilon_{c}$ \\
\hline \multirow{2}{*}{ Cover } & carbon fiber & 1840 & 37049 & 37049 & $\begin{array}{l}G_{12}=G_{13} \\
=2141\end{array}$ & 345 & $1.05 \%$ \\
composite & & & & $\begin{array}{l}G_{12}=G_{13} \\
=2141\end{array}$ & 345 & $1.05 \%$ \\
Carrying & $\begin{array}{c}\text { carbon fiber } \\
\text { Tensor }\end{array}$ & 1840 & 37049 & 37049 & $\begin{array}{l}G_{12}=G_{13} \\
=1409\end{array}$ & 425 & $3.2 \%$ \\
\hline \hline
\end{tabular}

Fig.5 The FE models of (a) solid plate, (b) honeycomb-core sandwich, and (c) tensor skin.

The FE models of three different plates: solid plate, honeycomb-core sandwich plate and the tensor skin, are depicted in Fig.5.The tensor skin comprised of cover ply, tensor ply and carrying ply, has the equal thicknesses of three plies, $h_{1}=h_{2}=h_{3}=1 \mathrm{~mm}$. The material of structure is assumed to be elastic-plastic material, and the Euler domain is assumed to be ideal fluid. The material properties of tensor skin are listed in Table 1.

Table 1 The material properties of tensor skin $[2,12]$

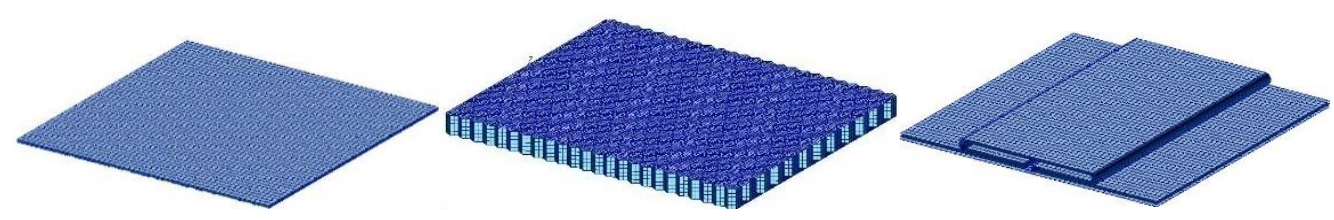

(a)

(c) 
transverse directions, respectively; $G$ is the shear modulus, $\mu_{12}=\mu_{13}=0.34$ are Poisson ratios; $\varepsilon_{c}$ is the fracture strain.

The total mass of the tensor skin, solid plate and honeycomb-core sandwich are adjusted to be the same. The solid plate that has thickness of $7 \mathrm{~mm}$, is made of the same material as the tensor ply listed in Table 1. The top and bottom surfaces of the honeycomb-core sandwich plate are the same material as the cover and carrying plies of the tensor skin, as listed in Table 1 and both have thickness $2.5 \mathrm{~mm}$. The thickness of the honeycomb core is $25 \mathrm{~mm}$, and core density is $184 \mathrm{Kg} \cdot \mathrm{m}^{-3}$ [18-20].

Around each plate, the same frame made of mild steel with density $7800 \mathrm{Kg} / \mathrm{m}^{3}$ is built, which constrains the displacement in the $x$ and $y$ direction, and the rotations in all the $x, y, z$ directions.

\subsection{Model in the Euler domain}

The Euler domain can be divided into two parts, i.e., air and water. The density of air is $1.24 \mathrm{Kg} / \mathrm{m}^{3}$ with specific internal energy $1.9 \times 10^{5} \mathrm{kcal} / \mathrm{kg}$ and $\gamma=1.4$. The volume of air is assumed to be $10 \times 10 \times 2 m$ as shown in Fig.6(a). The volume of the water being impinged is $10 \times 10 \times H$, where $H$ is the depth of water, which varies in computing cases. The water is assumed to be ideal fluid with density $1000 \mathrm{Kg} / \mathrm{m}^{3}$. The region of water is discretized by solid elements (CHEXA in DYTRAN) as shown in Fig.6(a). On all the 4 side surfaces parallel to the impact direction, non-reflective free boundary conditions are applied; while the bottom surface is fully constrained. 


\section{$4 \quad$ 3.3 Definition of fluid-structure interaction} on the coupling surface and distort the Lagrangian elements. terminate the simulation.

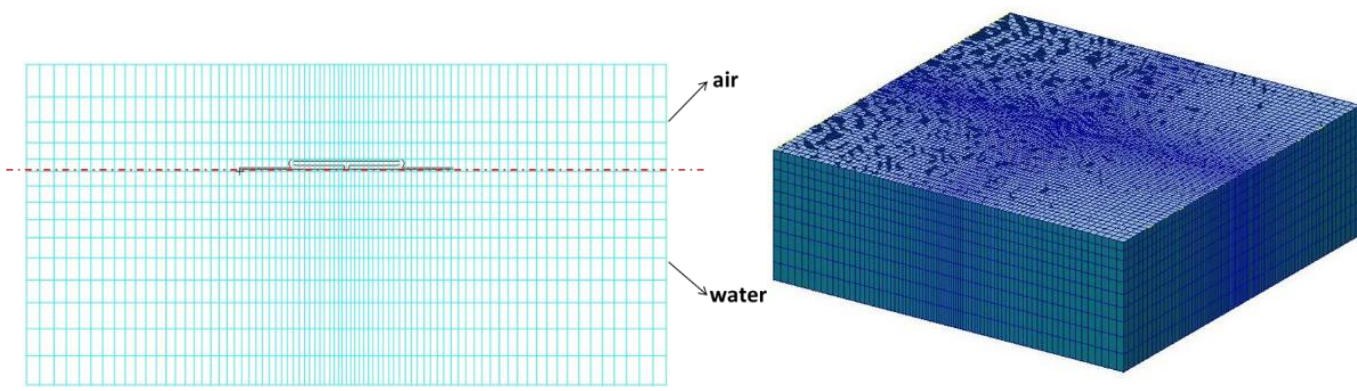

(a) The whole calculation region and (b) the water region

Fig.6 The FE model of Euler domain

The contact between the impinging plates and the water is defined according to the coupling surfaces with interactive failure [21].The aim of considering the fluid-structure interaction by using the coupling algorithm is to enable the objects modeled in Eulerian and Lagrangian meshes to interact. Here, the solid structure is discretized by Lagrangian meshes and the water is discretized by Eulerian meshes. The first task in coupling is used to transfer the forces between the two solver domains. In the same time, the stresses in the Eulerian elements produce forces to act

The coupling surface must form a closed and positive volume to meet DYTRAN's internal requirements [21]. In order to create a closed volume, it is necessary to add dummy shell elements. Since the additional grid points created for the dummy elements do not move as they are not connected to any structural elements, it should be large enough to cover the whole deformation range of the structure; otherwise the negative volume produced by large deformation would

It is not necessary to build additional coupling surface for the honeycomb-core sandwich plate, because its' top surface, bottom surface and cell wall of the core have already formed a 
closed volume. However, for the tensor skin and the solid plate, dummy shell elements need to be

2 created to get a closed volume. By taking the tensor skin as an example, the definition of the coupling surface is illustrated as follows. The height of the coupling surface adopted is larger than the maximum deflection of tensor ply, as shown in Fig.7. Meanwhile, at least one element must intersect with the Eulerian elements to transfer the forces between the two domains.

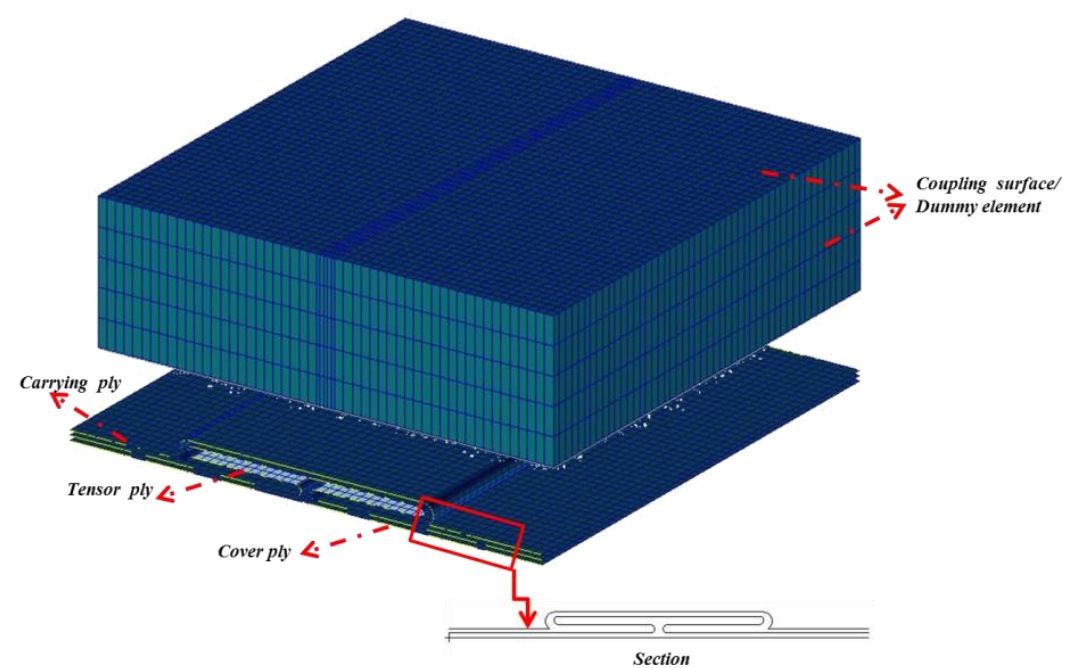

Fig.7 The definition of the fluid-structure interaction for the tensor skin

In the previous theoretical analysis, the central deflection of the tensor skin, which is fully clamped and subjected to pressure, is predicted. In the current FE model, the plate is supposed to impinge the water with a given initial velocity $v_{0}$. In order to make the results of FEM and theoretical predictions comparable, the relative deflection is defined as $u_{r}=u_{m}-u_{b}$, and the corresponding normalized deflection is defined as $\bar{u}=u_{r} / l$, where $u_{m}$ is the displacement of the plate center and $u_{b}$ is the displacement of the boundary, as shown in Fig.8.

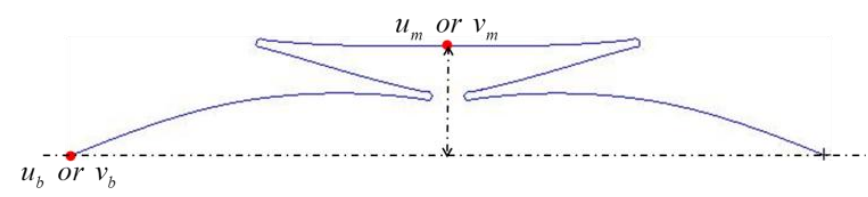

Fig. 8 The definition of the normalized central deflection 
1

\section{Comparison between three kinds of plates}

The dynamic responses of three kinds of plates, i.e., solid plate, honeycomb-core sandwich plate and tensor skin with the same length and width $1.5 \mathrm{~m}$, are compared with each other. Since all the three plates have the same total mass, $18.45 \mathrm{Kg}$, the same initial velocity will results in the same initial momentum. The ratio of the water depth to the plate length is $H / l$. Here $H / l=5$ is firstly adopted and the tensor skin with $k=0.5$ is taken as an example in calculation. Three different initial velocities, $v_{0}=10,15,20 \mathrm{~m} / \mathrm{s}$, are taken into account. DYTRAN can export the interaction pressure, which acts on the Eulerian elements intersecting with coupling surface. It is an average value considering the overall effect.

The deformation profiles of three different plates with same initial momentum, $I=276.75 \mathrm{Ns}$, are depicted in Fig.9. As seen from this figure, the solid plate is damaged shortly after impact, whilst no damage takes place in the tensor ply of the tensor skin.

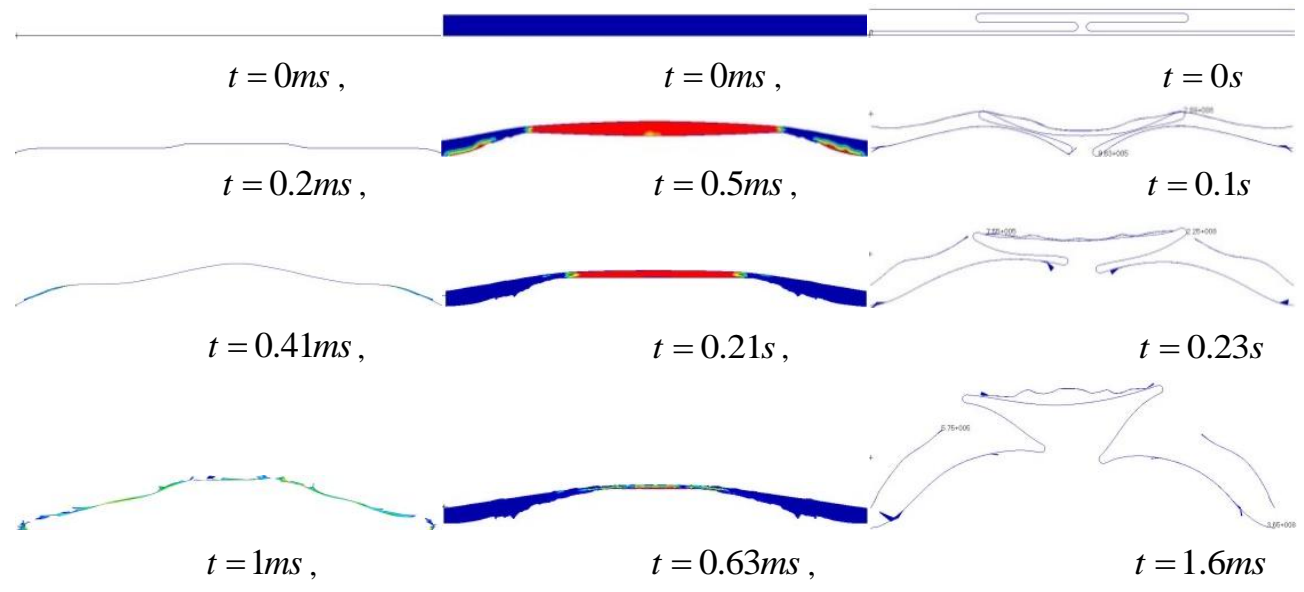

(a) Solid plate (b) Honeycomb-core sandwich(c) Tensor skin

Fig.9 The deformation profiles of three plates at different time instants ( $I=276.75 \mathrm{Ns}$ )

The normalized central deflection for different plates are shown in Fig.10, for the same initial momentum $I=184.5 \mathrm{Ns}$. As demonstrated in this figure, the tensor skin has the largest central 

the sandwich plate leads to a small central deflection, $\bar{u}=0.033$. The solid plate has the smallest one, $\bar{u}=0.004$, since its deformability is limited before the rapid breakage after the water impact.

4 The solid plate is safe for the applied momentum smaller than $138.38 \mathrm{Ns}$, while for the honeycomb-core sandwich plate and tensor skin, the critical momenta are 369Ns and 664.2Ns, respectively.

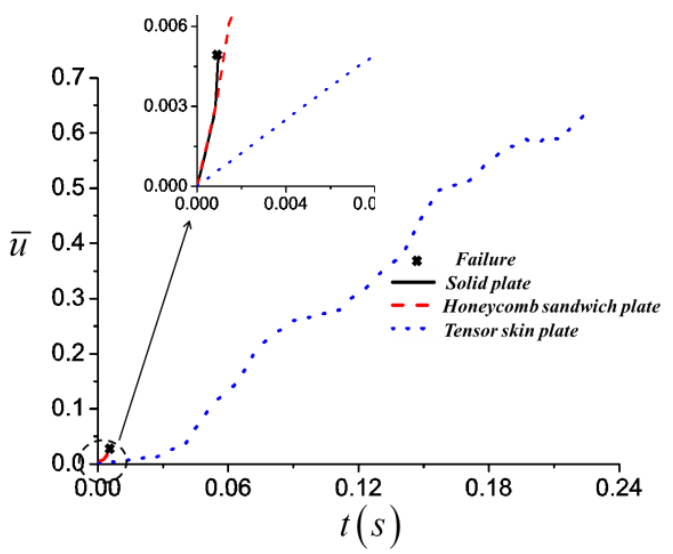

Fig.10 The normalized central deflection ( $I=184.5 \mathrm{Ns}$.)

The interaction pressure between water and plates are shown in Fig.11. Clearly, the intensity of the impact increases with the initial impact velocity for all three kinds of plates. For the solid plate, as illustrated in Fig.11(a), after the instant of the first contact to water, the interaction pressure first increases and then decreases rapidly. The pulse shape is very close to a triangular one. For the honeycomb-core sandwich plate, as shown in Fig.11(b), a short plateau of interaction pressure appears after the contact, and the pulse shape is not far from a rectangular one. Since the honeycomb-core sandwich absorbs energy mainly by the core's compression, the peak value of interaction pressure is clearly reduced. The plateau of the interaction pressure is also found in the pulse generated by the tensor skin, as shown in Fig.11(c), which is much lower and longer though. The pulse shape for the tensor skin is more like a trapezium pulse. This phenomenon can be 
1

2 3 4 5

explained as follows.The cover ply and the carrying ply break immediately after impact with water, and then the unfolding of tensor ply significantly reduces the peak of the contact pressure, while extends the pulse duration in the same time.
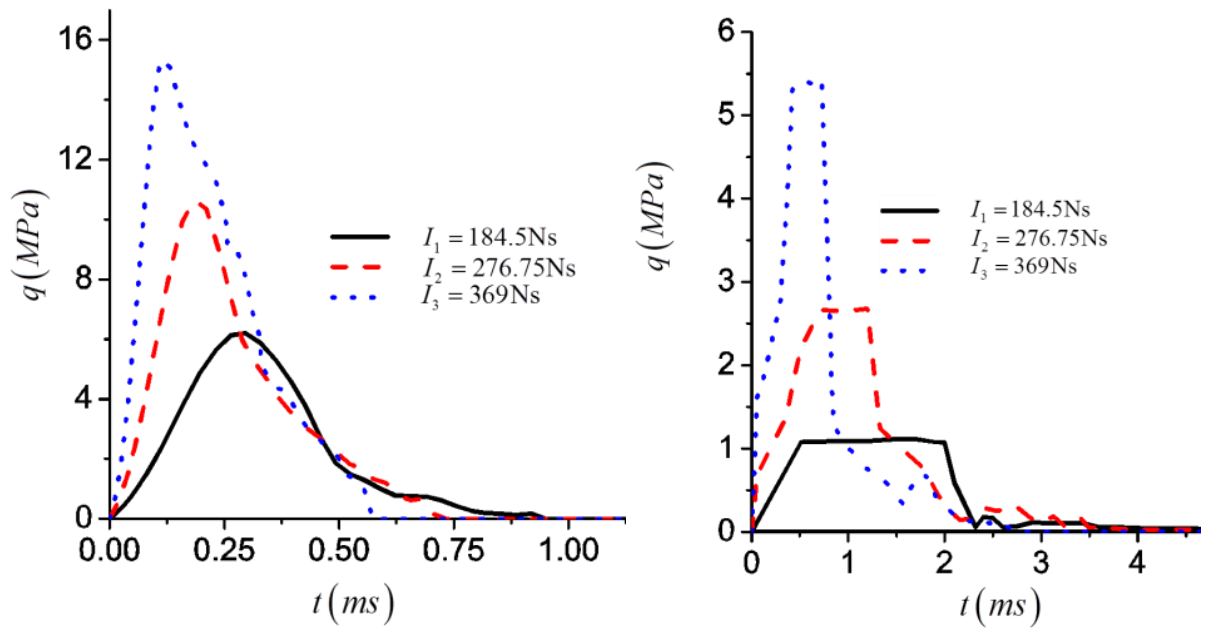

(a) Solid plate (b) Honeycomb-core sandwich
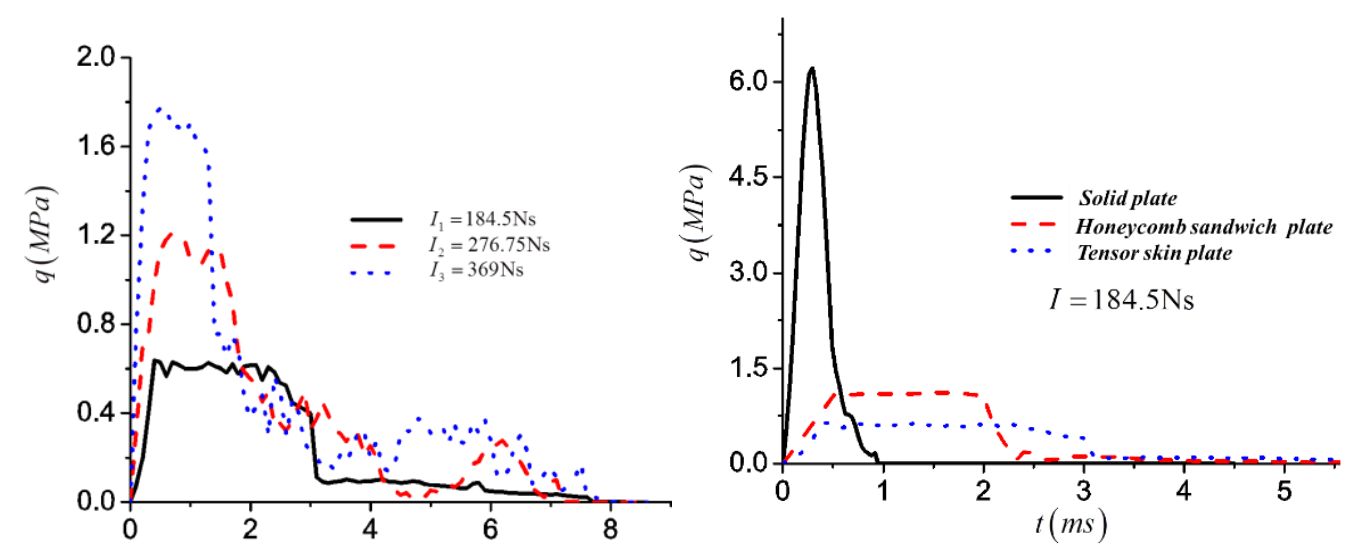

(c) Tensor skin(d) Comparison between three plates

Fig.11 The interaction pressure between the plate and water

The interaction pressure of three kinds of plates are compared in Fig.11(d), for $I=184.5 \mathrm{Ns}$. The peak pressures of the honeycomb-core sandwich plate and tensor skin are only $33 \%$ and $25 \%$, respectively, of that of the solid plate, whilst the pulse duration varies just in the reversed manner.

From the above comparison, it is concluded that the interaction pressure between the water and the plate depends on the structure configuration due to the effect of the fluid-structure 
interaction. Therefore, the fluid-structure interaction has to be adequately considered in the subsequent analysis of the dynamic response of various kinds of structures to water impact.

3 Compared with the solid plate, the impact pulses applied to the honeycomb-core sandwich and the

4 tensor skin are more moderate, as shown in Fig.11(d), which is good for their energy absorption.

5 Obviously, the energy absorbing capacities of the tensor skin and the honeycomb-core sandwich plate are both superior to the solid plate of the same mass, especially when the medium interaction

7 is considered. It should be noted that there is almost no air existing between the plate structure and

8 water during the impact process, as discussed in paper [17].

\section{$9 \quad 5$. Effects of the water depth}

Previous studies indicated that an impact on water would produce almost the same effect to an ordinary structure as an impact on a hard surface if the water is deep enough [22]. So the effect of water depth should be investigated. In this section, first the distribution of particle velocity along the water depth direction is investigated for a rigid plate impinging on water. Secondly, the dynamic responses of the three kinds of plates will be compared with each other when they impinge water of different depth.

\subsection{Rigid plate impinging on water}


5 during the time stage of $0 \sim 0.02 \mathrm{~ms}$. The particle velocities at different distance below the water bottom at about $0.05 \mathrm{~ms}$.

Fig.12 The interaction pressure of a rigid plate impinging on water with different depth

As shown in Fig.12, the peak of the interaction pressure increases with water depth and the time duration decreases with water depth. No reflection from bottom surface has been observed surface $h^{\prime}$ are plotted in Fig.13. Clearly, after impact, a disturbance is propagating along the depth direction in the water. In the same time, the intensity of the disturbance is decreasing since the peak of the particle velocity decreases with $h^{\prime}$. At the end of the impact pulse, i.e., $0.01 \mathrm{~ms}$ for $H=5 \mathrm{~m}$ as shown in Fig.12, the disturbed water depth is about $0.7 \mathrm{~m}$. The disturbance will reach the
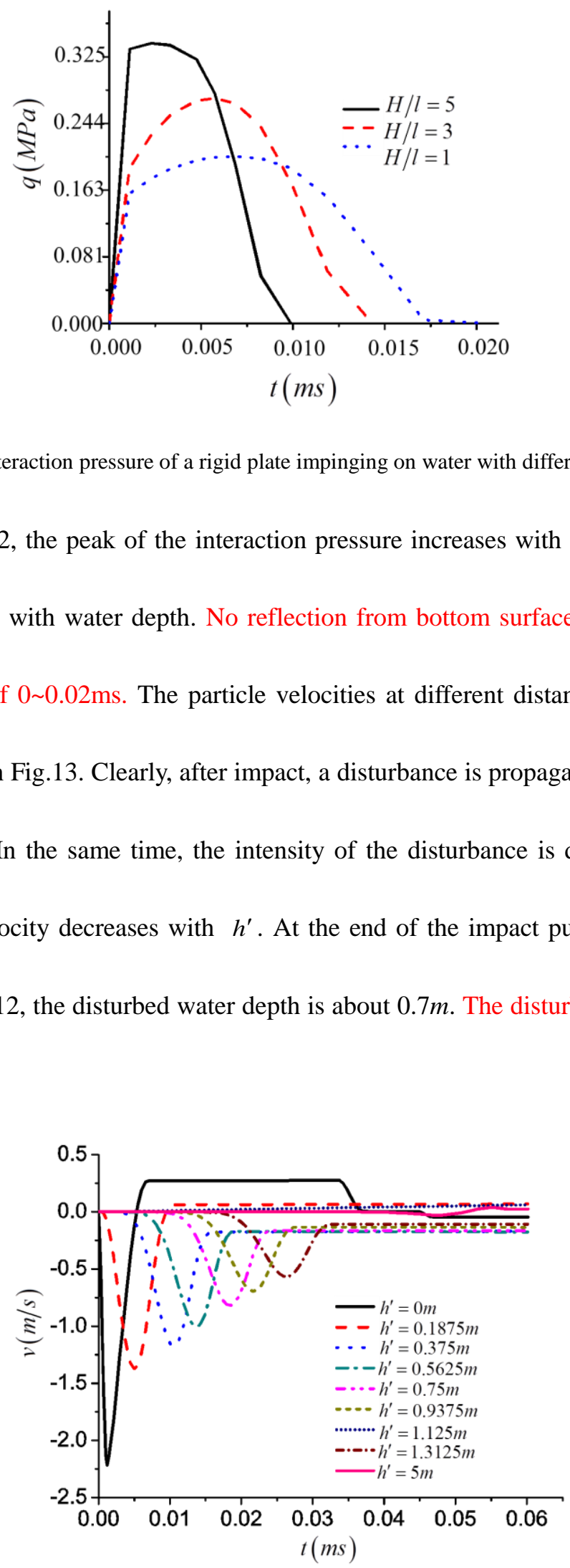
1

2

\subsection{Solid plate, honeycomb-core sandwich and tensor skin impinging on water}

Suppose all three different plates have the same length and width $1 m$, the same mass and initial velocity $20 \mathrm{~m} / \mathrm{s}$, thus the same initial momentum $195.2 \mathrm{Ns}$. The central deflections of different plates impinging on water of different depth $H / l$ are plotted in Fig.14.

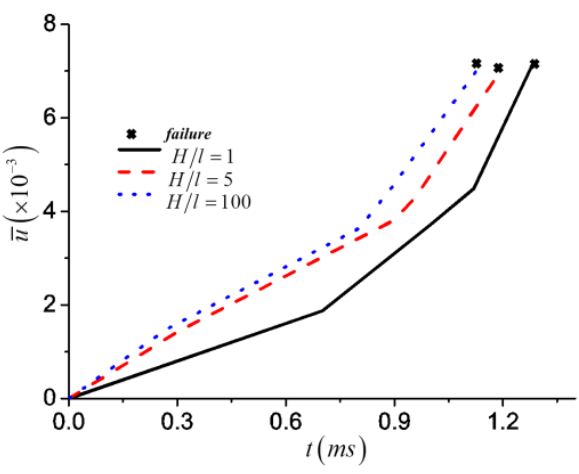

(a) Solid plate

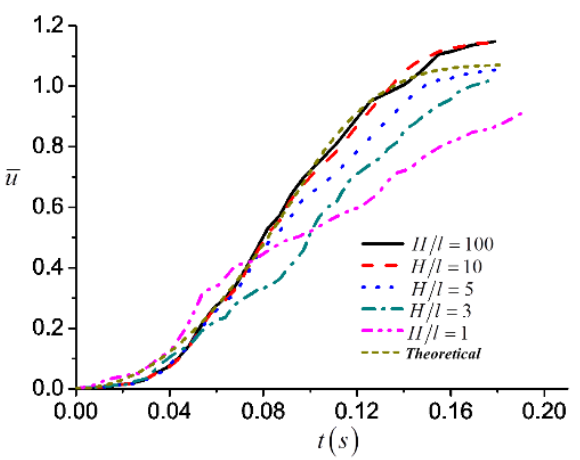

(c) Tensor skin $(k=0.5)$

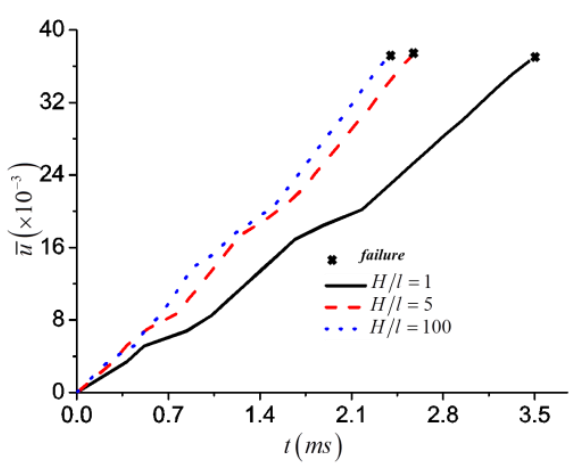

(b) Honeycomb-coresandwich

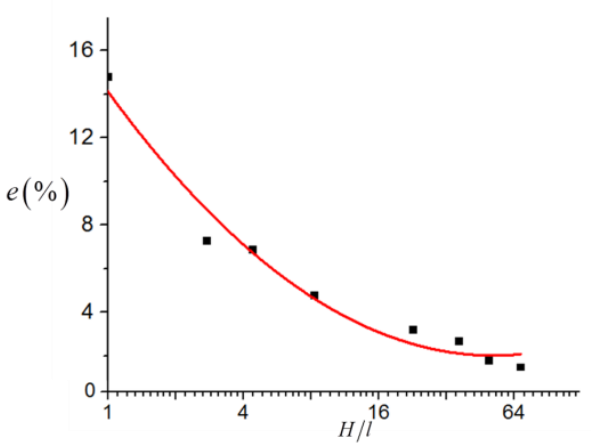

(d) Prediction errorfor tensor skin

Fig.14 The effect of water depth on the normalized center deflection

As shown in Fig.14, the solid plate and the honeycomb-core sandwich are all damaged after impinging on water of the current depth. Their final central deflection is independent from the water depth, and the total response time decreases with water depth. In comparison, the tensor skin is not damaged after impact, and its final deflection increases with the water depth. For water of low depth, that is low $H / l$, curves are fluctuating with time which is estimated to be caused by 
the reflection waves from the bottom. While the responses of these plates after impinging on deep water of $H / l \geq 5$ tend to agree with the respective theoretical predictions. Compared with the FE simulation, the errors in the central deflection predicted by the theoretical models decrease with the water depth, as shown in Fig.14(d). It is smaller than $8 \%$ for $H / l=5$.

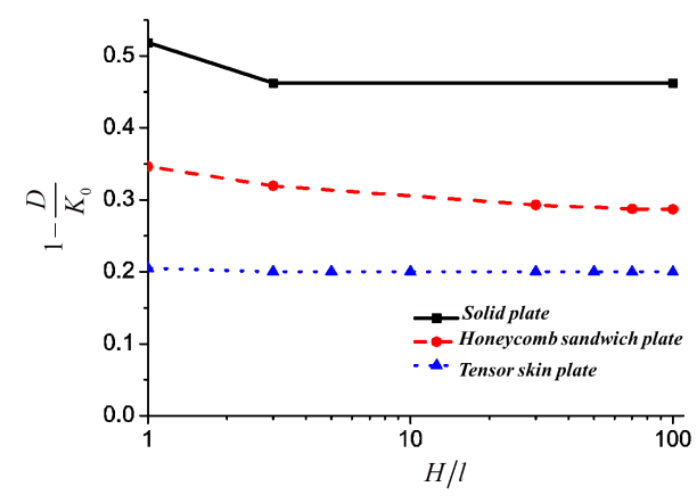

Fig.15 The energy transferred to water affected by water depth

Fig. 15 shows that the proportion of the total energy transferred to the water is affected by water depth. Here the energy transferred to the water is calculated by initial kinetic energy $K_{0}$ minus the plastic dissipation of the plate $D$. As demonstratedin this figure, the energy transferred into water by the tensor skin is smaller than the other two plates. The proportion of the energy acquired by deep water is found to be $46.2 \%, 28.9 \%$ and $19.9 \%$ for the solid plate, the honeycomb-core sandwich and the tensor skin, respectively. Again the plastic energies dissipated by the tensor skin and the honeycomb-core sandwich are larger than that of the solid plate.

\section{Verification of the theoretical predictions for tensor skin}

In the theoretical analysis of tensor skin[13], the water impact pulse was simplified as a rectangular pulse or a triangular pulse. In order to verify the theoretical predictions, the dynamic response of the tensor skin impinging on water is simulated by the fluid-structure interaction FE model, as well as the pulse loading FE models, which do not consider the fluid-structure 
interaction. two cases, $m v_{0}=I$. Here the water depth is taken as $H / l=5$.

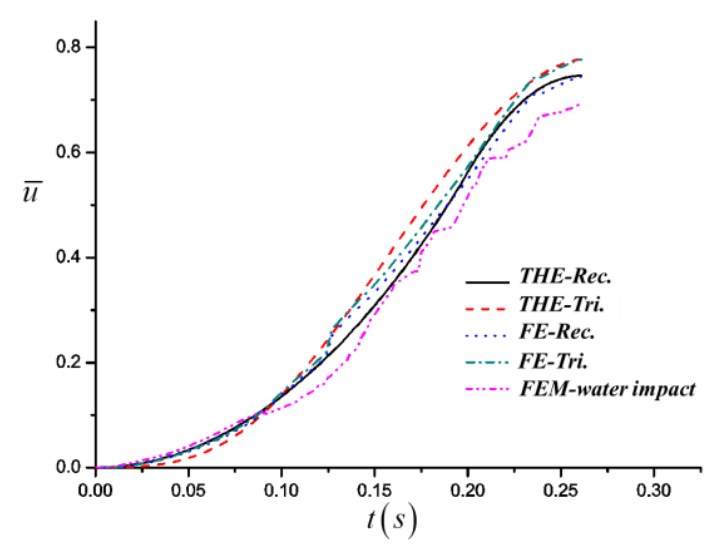

(a) Normalized central deflection

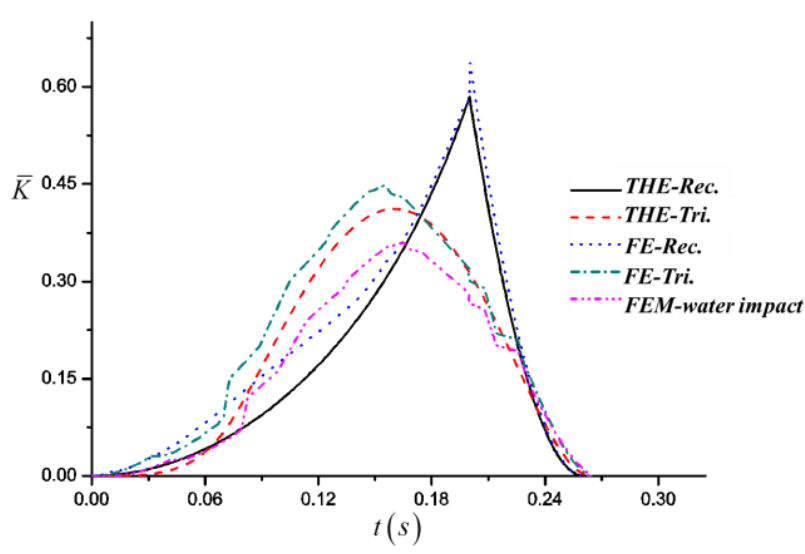
impulse is $I=240.1 N s$. to the above mentioned rectangular pulse and triangular pulse in the theoretical predictions.

(b) Normalized kinetic energy

Theoretical prediction: The tensor skin with $k=0.5$, width and half length $l=1$, total mass $18.45 \mathrm{Kg}$ is studied here. With a triangular pulse and a rectangular pulse $\left(\bar{q}=1.3 \bar{q}_{s 0}\right.$ and $\bar{t}_{d}=1$ ) are respectively applied, the results are shown in Fig.4. The corresponding value of this

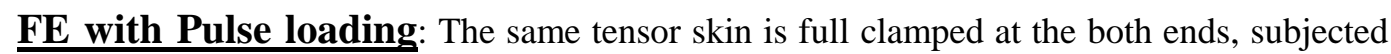

$\underline{\text { FE with Fluid-Structure interaction: }}$ The same tensor skin impinges water with initial velocity of $v_{0}=13 \mathrm{~m} / \mathrm{s}$, where the initial momentum is equal to the applied impulse of the above 
The dynamic responses predicted by above three models are compared in Fig.16. The normalized central deflection and the kinetic energy $\bar{K} \equiv K / M_{p}$ obtained by FEM simulations

4 are all lower than that by the theoretical predictions, but the discrepancies are all small. In the theoretical predictions, the total impulse applied is taken as $I=m v_{0}$, that is, it is equal to the initial momentum of the plate in the interaction FE model. In fact, the total initial momentum is transferred to the plate as well as the water, which could be confirmed by the energy conservation,

8 as shown in Fig.15. However, the deviation of about 10\% indicates the simplified theoretical model is acceptable.

\section{Conclusions}

In this paper, in order to verify the previous theoretical prediction of tensor skin under water impact, the fluid-structure interaction models are constructed for a solid plate, a honeycomb-core sandwich plate and a tensor skin. interaction pressure generated by the solid plate is recognized as a triangular pulse; and those generated by the honeycomb-core sandwich and the tensor skin are close to arectangular pulse and a trapezium pulse, respectively. Compared with the solid plate, the loading pulses on the honeycomb-core sandwich plate and the tensor skin are more moderate, and their energy absorbing capacities are confirmed to be superior to the solid plate of the same mass. tensor skin are appropriate for deep water case. The final deflection obtained by the FE model 
with fluid-structure interactionis smaller than those given by the pulse loading FE model and the

\section{Acknowledgements} fluid-structure interaction.

\section{References}

theoretical prediction, while the difference is estimated as $10 \%$. Thereby, the theoretical model and the pulse approximation method are verified by the FE simulation with a consideration of the

The authors gratefully acknowledge the financial support from the National Science Foundation of China No.11372163, the National Fundamental Research Program of China through Grant No.2010CB832701 and No.2011CB610305. The $3^{\text {rd }}$ author (T.X. Yu) would like to thank the support from the NSFC Key Project No. 11032001.

1. Thuis, H.G.S.J. and Wiggenraad,J.F.M., A tensor skin concept for crashworthiness of helicopters in case of water impact. 1994, National Aerospace Laboratory NLR.

2. Labeas, G. and Kermanidis, T.H., Crushing behavior of the energy absorbing 'tensor skin ' panels. Fatigue Fract Engng Mater struct 2003. 26: p. 449-457.

3. Thuis, H., Vries,H. and Wiggenraad, J., Subfloor skin panels for Improved Crashworthiness of Helicopters. 1999, National Aerospace Laboratory NLR. p. 1-16.

4. Michielsen, A.L.P.J., Wiggenraad, J.F.M., Ubels, L.C., Frijns, R.H.W.M., Kohlgrüber, D., Labeas, G. and McCarthy, M.A., Design, Test and Analysis of Tensor Skin Panels for Improved Crashworthiness in Case of Water Impact, in the AHS Crashworthiness Specialists' Meeting "Crash Safety Challanges and Innovative Solutions". 1998, National Aerospace Laboratory: Phoenix, Arizona. p. 1-10.

5. Ubels, L.C., Johnson, A.F., Gallard, J.P. and Sunaric, M., Design and testing of a composite bird strike resistant leading edge, in The SAMPE Europe Conference \& Exhibition. 2003, National Aerospace Laboratory Paris, France. p. 1-16.

6. Huis, H.G.S.J., Vries, H.P.J.D., and Wiggenraad, J.F.M., Sub-floor skin panels for improved crashworthiness of helicopters in case of water impact. in Annual Forum Proceedings American Helicopter Society, v 1. 1995.

7. McCarthy, M.A. and Wiggenraad, J.F.M., Numerical investigation of a crash test of a composite helicopter sub_floor structure. Composite Structures, 2001. 51: p. 345-349.

8. Kermanidis, Th., Labeas, G., Apostolopoulos, C. and Louis Michielsen, Numerical simulation of composite structures under impact. International Conference on Structures Under Shock 
and Impact, 1998. 32: p. 591-600.

9. McCarthy, M.A., Harte, C.G., Wiggenraad, J.F.M., Michielsen, A.L.P.J., Kohlgrüber, D. and Kamoulakos, A., Finite element modeling of crash response of composite aerospace subfloor structures. Computational Mechanics, 2000. 26: p. 250-258.

10. Labeas, T.K.G. and Ubels, M.S.L., Development and Validation of a Novel Bird Strike Resistant Composite Leading Edge Structure. Applied Composite Materials, 2005. 12: p. 327-353.

11. Kermanidis, Th, Labeas, G., Sunaric, M., Johnson, A.F. and Holzapfel, M., Bird strike simulation on a novel composite leading edge design. International Journal of Crashworthiness, 2006. 11(3): p. 189-202.

12. Mi, B.W. and Zhao, M.Y., Analysis of anti-bird strike for composit material tensor skin wing structures. Computer Simulation, 2010. 27(2).

13. Ren, Y.T., Qiu, X.M. and Yu, T.X., The sensitivity analysis of a geometrically unstable structure under various pulse loading. International Journal of Impact Engineering, 2014. 70: p. 62-72.

14. Ren, Y.T., Qiu, X.M. and Yu, T.X., Theoretical analysis of the static and dynamic response of tensor skin. International Journal of Impact Engineering, 2014. 64: p. 75-90.

15. Youngdahl, C.K., Influence of pulse shape on the final plastic deformation of a circular plate. International Jounal of Solids Structure, 1971. 7: p. 1127-1142.

16. Li, Q.M. and Jones, N., Foundation of correlation parameters for eliminating pulse shape effects on dynamic plastic response of structures. American Society of Mechanical Engineers, 2005. 72: p. 172-176.

17. Bin, X., Zhen, C. and Xi, X., Thesimulationanalysisoftheslammingofelasticflat-bottom marine structure. China Offshore Platform, 2005. 20(1): p. 22-28.

18. Yi, T. and Chen, C.Q., The impact response of clamped sandwich beams with ordinary and hierarchical cellular cores. International Journal of Impact Engineering, 2012. 47: p. 14-23.

19. Qiu, X., Deshpande, V., and Fleck, N., Finite element analysis of the dynamic response of clamped sandwich beams subject to shock loading. European Journal of Mechanics-A/Solids, 2003. 22(6): p. 801-814.

20. Tang, Y. and Xue, M., Thermo-mechanicalcharacteristics analysis of sandwich panel with honeybomb core. Acta Materiae Compositae Sinica, 2005. 22(2): p. 130-136.

21. Dytran 2012 User's Guide. 2012.

22. Korobkin, A., Analytical models of water impact. European Journal of Applied Mathematics, 2004. 15(06): p. 821-838. 